\title{
Diseño de un protocolo de prácticas de enfermería en nefrología según la metodología ABP
}

\author{
$M^{\mathrm{a}}$ del Mar Rueda Jarque - Maria Cano Pulido - $\mathbf{M}^{\mathrm{a}}$ José Arnau Vives
}

Servicio de Nefrología. Hospital Vall d’Hebron. Barcelona

\section{Sra. Directora:}

En el Servicio de Nefrología del área general del Hospital Universitario Vall d'Hebron, que incluye Hospitalización, Trasplante Renal, Diálisis Peritoneal y Hemodiálisis, realizan prácticas clínicas estudiantes de $3^{\circ}$ de Enfermería de la Escola Universitària $\mathrm{d}^{\prime}$ Infermeria (EUIVH) de nuestro hospital. La EUIVH imparte sus estudios siguiendo la metodología ABP (Aprendizaje Basado en Problemas) desde el curso académico 2002-2003, para adaptarse al Espacio Europeo de Educación Superior ${ }^{1}$. EI ABP es un método de enseñanza-aprendizaje centrado en el estudiante, en el que éste adquiere conocimientos, habilidades y actitudes a través de situaciones de la vida real. Su finalidad es formar estudiantes capaces de analizar y enfrentarse a los problemas de la misma manera en que lo harán durante su actividad profesional ${ }^{2}$.

En las prácticas clínicas, el tutor es la enfermera asistencial que, en su servicio o unidad, guía el proceso de aprendizaje de los estudiantes y es un referente y un soporte pedagógico en este período de formación ${ }^{3}$. Es la encargada de facilitar la puesta en práctica de conocimientos, habilidades y actitudes para que el estudiante afronte de la manera más adecuada las situaciones emergentes en la práctica asistencial, además es quien evalúa el proceso de aprendizaje de prácticas del alumno.

Nuestro objetivo es diseñar un protocolo de prácticas clínicas que sirva de guía, tanto a la enfermera de referencia como a los estudiantes, a que éstos adquieran conocimientos, habilidades y actitudes en enfermería nefrológica, en consonancia con la metodología ABP.

\begin{tabular}{|c|}
\hline Correspondencia: \\
$M^{\text {a }}$ del Mar Rueda Jarque \\
Servicio de Nefrología \\
Hospital Vall d'Hebron \\
$\mathrm{P}^{\circ}$.Vall d'Hebron, 119-129-08035 Barcelona \\
mjoarnau@hotmail.com
\end{tabular}

Formamos un grupo focal de discusión de 3 enfermeras, una enfermera tutora y de referencia de los alumnos en prácticas, otra enfermera también de referencia, y la supervisora del Servicio. Las dos enfermeras de referencia realizamos el curso para tutores de práctica clínica impartido por la EUIVH, con formación en la metodología ABP. Tras varias reuniones de trabajo se obtuvo un protocolo de prácticas clínicas, en el que estructuramos el periodo de prácticas en un cronograma, definiendo los períodos de tiempo que el estudiante estará en cada área de la unidad y los objetivos de aprendizaje a conseguir en cada una de ellas (tabla 1 ).

Este protocolo es entregado al alumno el primer día de las prácticas en nuestra unidad. Ellos buscarán la información sobre patología renal y cuidados de enfermería, que discuten con su enfermera de referencia y trabajan las habilidades y actitudes durante todo el período de prácticas.

Para evaluar la eficacia de este protocolo, pasamos un test de retroacción a realizar por las enfermeras de referencia, para determinar si nos ayuda a mejorar nuestro rol docente. A los estudiantes se les pasa un cuestionario sobre conocimientos teóricos, aparte de la evaluación que se realizará de forma transversal, mediante observación directa y preguntas abiertas, siguiendo los objetivos del protocolo y los del documento evaluativo de la EUIVH .

Somos conscientes que al finalizar las prácticas los estudiantes no serán expertos en Nefrología, especialmente en lo que se refiere a las técnicas, tanto de diálisis peritoneal como de hemodiálisis, pero si queremos lograr que conozcan los aspectos más relevantes de la enfermedad renal. Sabemos la dificultad de tener enfermeros expertos en el campo de la nefrología, y últimamente la falta de profesionales. Realizando este tipo de prácticas que engloba todos los campos de la nefrología el estudiante puede conoce en profundidad los cuidados enfermeros en nefrología y sabrá al finalizar sus estudios si realmente quiere profundizar en dicha especialidad. 


\section{Bibliografía}

1. Disponible en http://www.vhebron.es/do/eui/home. htm

2. Bernabeu Tamayo MD, Consul Giribert M. Aprendizaje basado en problemas. En: Cónsul Giribert M, Montenegro Marchante L, Arreciado Marañón A, Bernabeu Tamayo MD, Bernaus Poch E,
Blanco Sanchez R, Branda L et al. Historia de un cambio: un currículum integrado con el aprendizaje basado en problemas. Escola Universitària d'Infermeria Vall d'Hebron. Barcelona 2007. p. 35-53.

3. Generalitat de Catalunya. Institut d'Estudis de la Salut. El tutor d'Infermeria: perfil, funcions i competències. 2003.

\section{HOSPITALIZACIÓN Y TRASPLANTE RENAL (TR) - 6 SEMANAS}

$1^{a}$ semana:

$\checkmark$ Conocer la dinámica del servicio: miembros del equipo asistencial, rutinas/circuitos del servicio, registros de enfermería.

$\checkmark$ Adquirir conocimientos sobre la patología renal: anatomía y fisiología, enfermedades más frecuentes (IRA, IRC, GN, HTA) y sintomatología, medicación.

$2^{\mathrm{a}}$ a $5^{\mathrm{a}}$ semana:

$\sqrt{ }$ Adquirir conocimientos y habilidades en cuidados de enfermería nefrológica: cuidados generales, accesos vasculares de HD, pruebas diagnósticas, pre y postoperatorio del TR, educación sanitaria.

$\checkmark$ Realización de un PAE.

$\checkmark$ Trabajar los conocimientos, habilidades y actitudes con el paciente renal.

$6^{\mathrm{a}}$ semana:

$\sqrt{ }$ Evaluación formativa del estudiante. Autoevaluación.

\section{DIALISIS PERITONEAL (DP) - 2 SEMANAS}

$1^{\text {a }}$ semana:

$\checkmark$ Conocer la dinámica de la unidad.

$\sqrt{ }$ Adquirir conocimientos sobre la DP: concepto, indicaciones y contraindicaciones.

$\checkmark$ Conocer el material necesario para la técnica.

$2^{\mathrm{a}}$ semana:

$\sqrt{ }$ Adquirir habilidades en DPCA y DPA: conexión y desconexión del paciente, montaje de la cicladora.

$\checkmark$ Trabajar los conocimientos, habilidades y actitudes en el seguimiento de la terapia del paciente y educación sanitaria.

\section{HEMODIALISIS (HD) - 2 SEMANAS}

$1^{a}$ semana:

$\sqrt{ }$ Conocer la dinámica del área de HD.

$\checkmark$ Adquirir conocimientos teóricos sobre la HD.

$\checkmark$ Conocer el material necesario para la realización de la técnica.

$2^{a}$ semana:

$\sqrt{ }$ Adquirir habilidades en el montaje y cebado del circuito extracorpóreo.

$\sqrt{ }$ Trabajar los conocimientos, habilidades y actitudes del estudiante con el paciente durante una sesión de HD.

$\checkmark$ Educación sanitaria.

Tabla 1. Protocolo de prácticas clínicas en Enfermería nefrológica según la metodología ABP 